\title{
Inferring intrinsic polarisation angles of radio sources from SKA observations
}

\section{J. Geisbuesch*, P. Alexander}

Astrophysics Group, Cavendish Laboratory, University of Cambridge

E-mail: joernemrao.cam.ac.uk

We discuss how SKA linear polarisation measurements can be used to learn about intrinsic geometries of background sources and, thus, their polarisation and Faraday depth. For this purpose, we consider source models of differing complexity. Moreover, we investigate the influence of Faraday foreground screens on the sources' polarisation. In particular, we focus on galaxy clusters, whose effects on the observed polarisation are modelled numerically. By applying statistical data analysis tools we investigate how well intrinsic source and cluster 'Faraday properties' can be recovered from SKA polarisation data on the basis of a factual model.

From Planets to Dark Energy: The Modern Radio Universe $1^{\text {st }}-5^{\text {th }}$ October 2007

The University of Manchester, United Kingdom

* Speaker. 


\section{Introduction}

A powerful tool for studying magnetic fields in a variety of environments is to use Faraday rotation against background polarised sources. This population of sources consists of mainly starforming galaxies and radio AGN. In order to analyse spectral polarisation data, we need to have a (general) parameterised model for the background source population. The model must allow for structure of polarised emission in the source together with intrinsic depolarisation. At the source a model depolarisation must include intrinsic Faraday dispersion and also depolarisation due to random field components. Between the source and the observer, we must consider not only the total Faraday depth, but also depolarisation due to structure in the Faraday foreground screen on scales smaller than the telescope resolution.

Note that, given the extent of the longest expected SKA baselines (up to several hundred kilometres), which provide arcsecond resolution for the frequency range of interest and allow resolution of turbulent regions on scales of the order of a kpc even at redshifts of $z \approx 1$, the beam depolarisation can be disregarded in most cases.

\section{Modelling galactic sources}

The vast majority of background sources detected by SKA observations will be
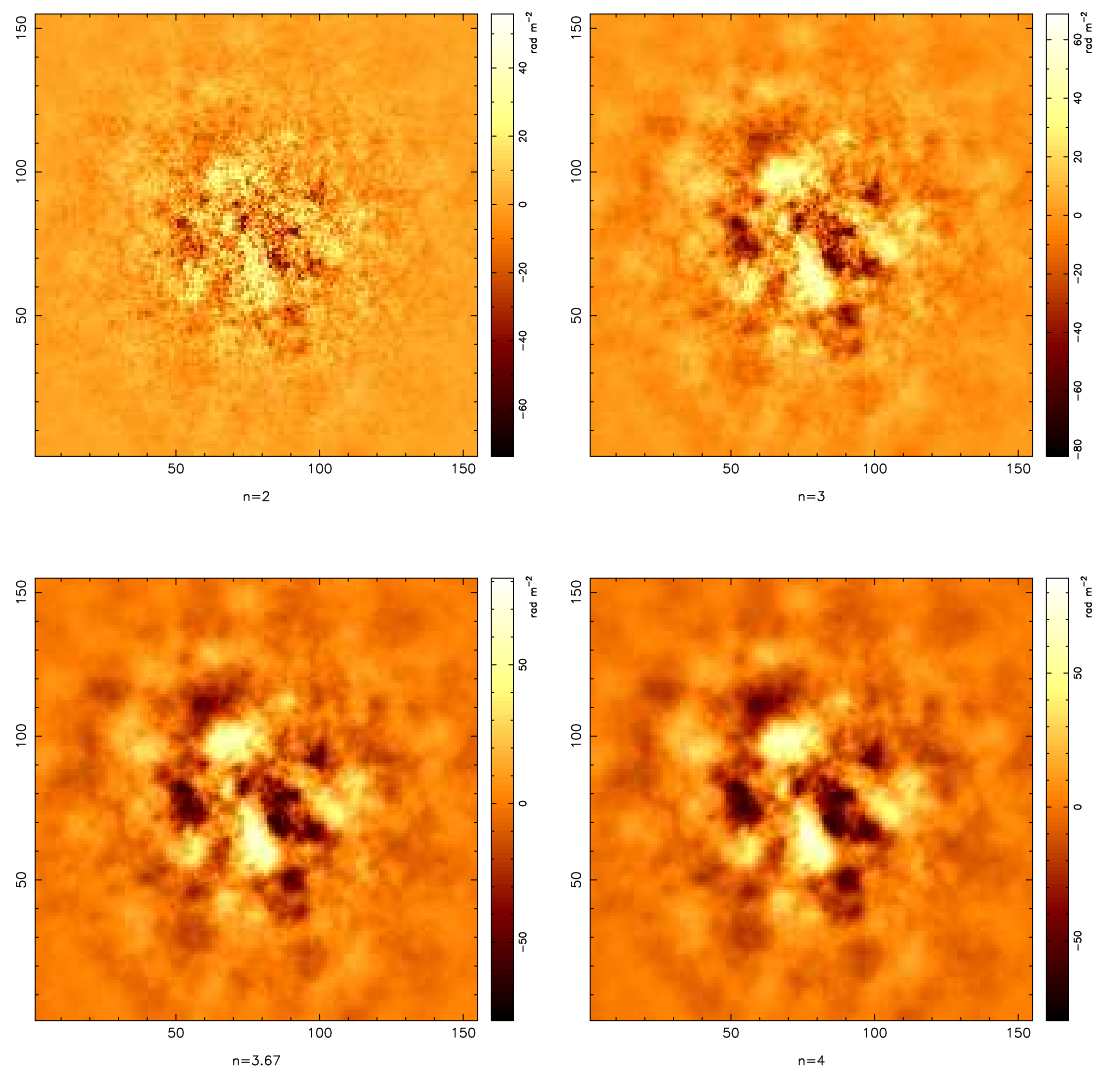

Figure 1: Simulated RM images of a medium mass medium redshift galaxy cluster for different values of the spectral index of the power spectrum of magnetic field fluctuations. spiral star-forming galaxies. Their magnetic field consists of a disk and a much weaker halo component, apart from some turbulent component. Dependent on the origin of the seed magnetic field, disks possess axis- and/or bisymmetric dynamo fields. The turbulent component arises from stellar activity and its magnitude can be of the order of the regular field one.

\section{Clusters}

\section{as Faraday}

\section{foreground screens}

Common magnetic field strengths in clusters are typically $0.1-1 \mu \mathrm{G}$. 
Cooling flow clusters have stronger magnetic fields than non-cooling flow clusters - up to an order of magnitude stronger. Radio images of clusters show halos and relics, which suggest that wide-spread magnetic fields reside in clusters (minimum energy calculations give $B \sim 0.4-1 \mu \mathrm{G}$ ).

A simple way to model

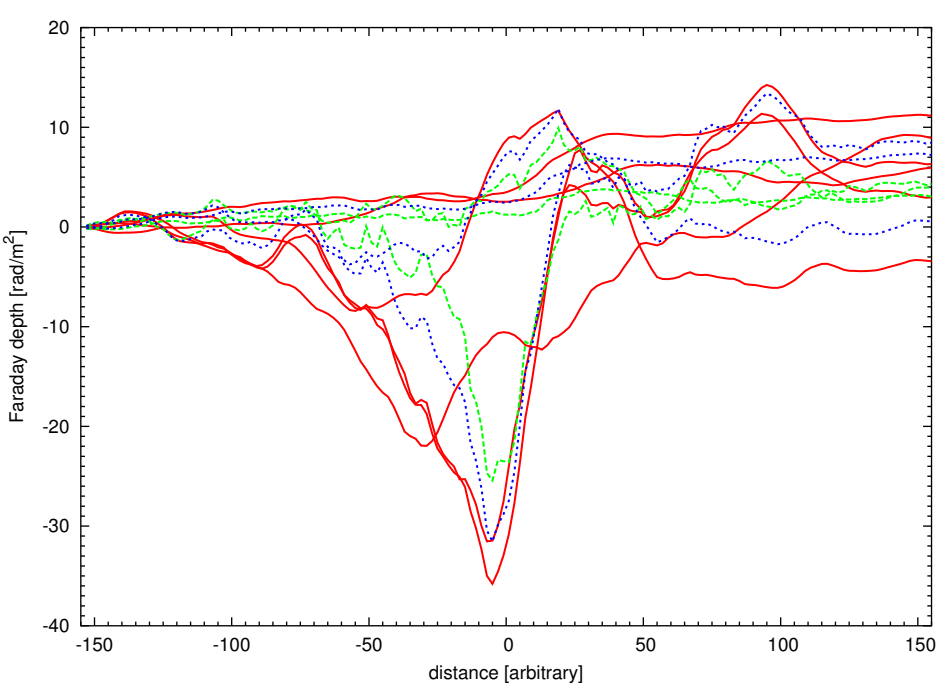

Figure 2: Change of the Faraday depth for different lines of sight through the cluster. The colours and line-styles indicate the spectral index of the cluster magnetic field power spectrum. cluster magnetic fields is to divide the cluster up into cells on whose scale the electron density and magnetic field vector can be regarded as uniform (see also [1]). Here, we adopt a power law spectrum to model correlations of the cell magnetic field vectors. For the electron density distribution we assume a conventional King profile and also scale the magnetic field strength in a similar fashion (i.e. decreasing with cluster radius).

Figure 1 shows cluster magnetic field realisations for different assumed spectral indeces of the cluster magnetic field power spectrum. The larger $n$ is, the less power resides in small scale fluctuations. Figure 2 shows how the Faraday depth changes along different lines of sight through a cluster.

\section{Rotation Measure grids}

Cluster X-ray in combination with RM observations can yield estimates of cluster magnetic fields. However, current RM grids are sparse and the sample of clusters which have a larger number of polarisation measurements of imbedded or background sources is small. The SKA with its unique collection area reaches unmatched sensitivities for reasonable integration times. Thus, the number of source detections within a cluster will significantly increase. It even might be that integration times will be limited by source confusion. Further discussion of the RM grid is given in Alexander et al. (this volume; see also [2]).

\section{Reconstructing intrinsic source and foreground Faraday screen properties}

To investigate the potential of future SKA data to determine (radio) source intrinsic and (cluster) foreground screen magneto-ionic properties, we model SKA mid band observations from 300 $\mathrm{MHz}$ to $1 \mathrm{GHz}$. Our telescope model is based on the most recent SKA reference design (see [3]). Our line of sight simulations assume radio sources at redshift $z=2.5$ which are 'Faraday shadowed' by a redshift $z=0.5$ galaxy cluster. Radio sources are modelled to possess a mixture of thermal and non-thermal electrons. Amplitudes of regular and random magnetic fields and their ratios are chosen in such way that the resulting degrees of polarisation are consistent with Stokes I, Q and U and 

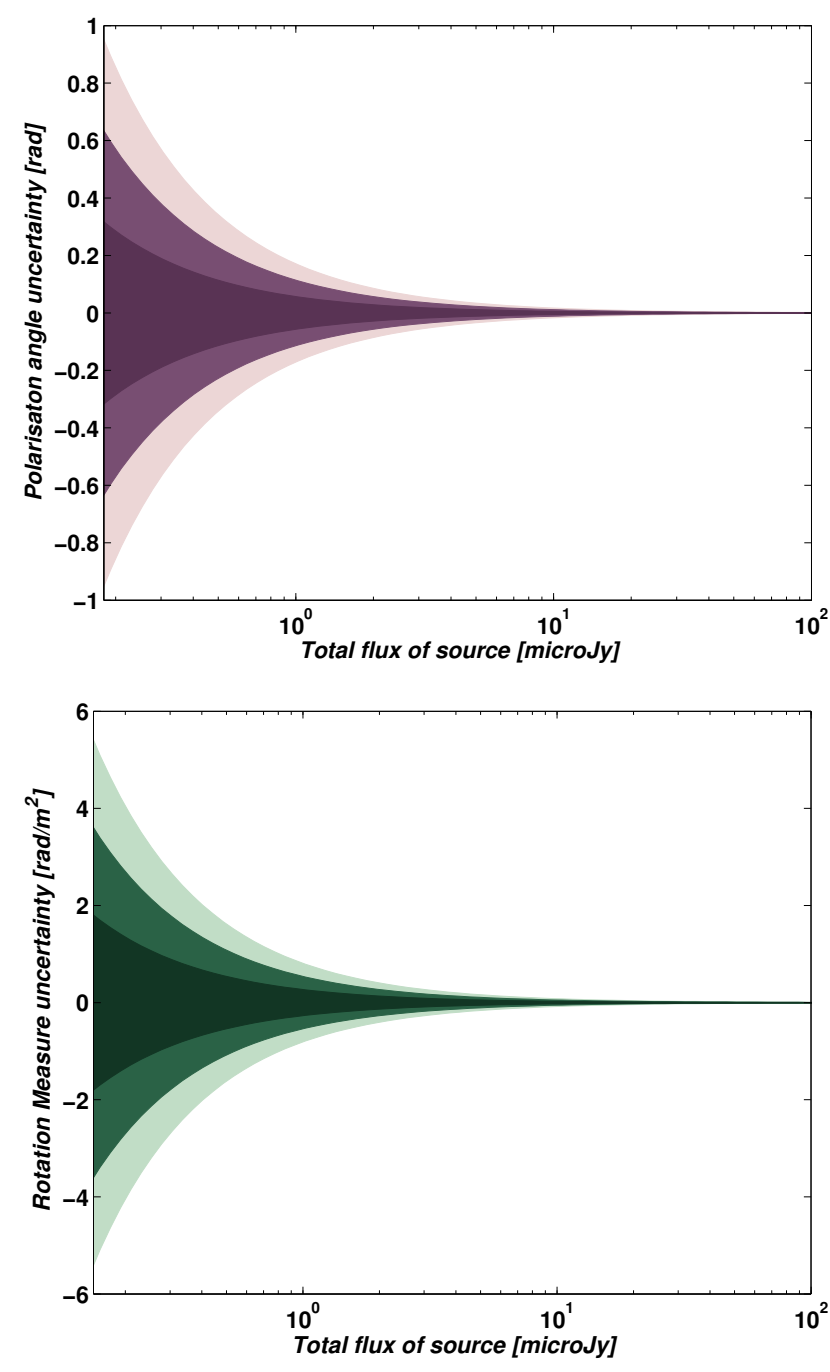

Figure 3: (a) Uncertainties on the reconstructed intrinsic source polarisation angle (b) and on the reconstructed RM of the foreground screen for a single line of sight observation simulation as described in the text. In each case the different colour shading indicates the 1,2 and $3 \sigma$ uncertainty.
RM observations of such sources. Hence, our modelled sources possess intrinsic RMs and the spectral behaviours of their polarisation degrees depend on their internal magneto-ionic structure. While sources can be regarded as unresolved, the cluster in the foreground has an angular diameter much larger than the SKA resolution. Its Faraday shadow is due to a turbulent magnetic field (see Figure 1 and section 3) imbedded in its thermal electron gas. However, it is assumed that the smallest spatial scale, on which the cluster turbulent field fluctuates (high- $k$ power spectrum cut-off), is larger than the size of the area illuminated by the source so that external depolarisation can be neglected. The telescope integration time assumed for generating the mock data is approximately 3 hours. Note further, that our modelling neglects disturbances due to Galactic foregrounds. To the simulated data, we apply a Bayesian analysis, which uses a Markov Chain Monte Carlo method to explore the posterior distribution and to evaluate how well 'Faraday properties' can be constrained by future SKA observations. Thereby, our analysis assumes that redshifts (of the two astrophysical objects) are a priori known to some realistic degree of precision as achievable by present X-ray and optical/infra-red facilities respectively. In Figure 3 reconstruction uncertainties on the inferred polarisation angle and foreground screen RM are shown for a particular radio source model as function of its total radio flux. The constraints are obtained by marginalising in each case over all other parameters.

\section{Conclusions}

Our simulations suggest that from SKA observations we expect to gain unmatched insights into intrinsic source properties as well as extract information about Faraday foreground screens. However, due to differing source intrinsic properties and the turbulent magnetic field component being dominant in clusters, dense RM grids are essential to explore the nature of such foreground screens. Concludingly, we note that the SKA and already its pathfinders will be extremely suitable telescopes to study the magnetic Universe and the cosmic evolution of magnetic fields. 


\section{References}

[1] Murgia M., Govoni F., Feretti L. et al., A\&A, 424, 2004, 429-446.

[2] Geisbuesch J., Alexander P., Krause M. \& Bolton R., A Century of Cosmology, Venice, August 2007.

[3] Schilizzi R. T. et al., Specifications for the Square Kilometre Array, September 2007. 